\title{
LOS DERECHOS DE LAS MUJERES INDÍGENAS DE LA COMUNIDAD DE TOCAGÓN DESDE EL PLURALISMO JURÍDICO
}

\author{
Zoila Guamán Amaguaña ${ }^{1}$, Pablo Ricardo Mendoza Escalante ${ }^{2^{*}}$, Ana Julia Romero González ${ }^{3}$ \\ ${ }^{1}$ Universidad de Otavalo, Facultad de Derecho, Otavalo, Ecuador. Abogada de la Universidad de Otavalo. \\ ${ }^{2}$ MSc en Desarrollo Agrario de la Universidad de Los Andes y Docente de la Universidad Otavalo. \\ ${ }^{3} \mathrm{PhD}$. En Ciencias humanas de la Universidad del Zulia. Venezuela. Docente investigadora de la Universidad de Otavalo.
}

*Autor para correspondencia: pmendoza@uotavalo.edu.ec

Recibido: 2021/04/08 Aprobado: 2021/05/31

D0I: https://doi.org/10.26621/ra.v1i24.679

\section{RESUMEN}

El presente trabajo de investigación tuvo como objetivo analizar desde el Pluralismo Jurídico, los derechos de las mujeres indígenas de la comunidad de Tocagón en relación con el Derecho Convencional. La investigación estuvo fundamentada en un enfoque cualitativo, de diseño descriptivo-documental. Como instrumento para la recolección de información se diseñó un cuestionario tipo encuesta, conformado por 11 preguntas de respuestas cerradas, dicotómicas. El mismo se aplicó a una muestra de 75 mujeres indígenas Kichwa de la comunidad de Tocagón, extraída de una población de 292. El análisis de resultados arrojó que un alto porcentaje de las encuestadas desconoce la existencia de normas nacionales e internacionales relacionadas con los derechos de las mujeres (65\%) y sobre conceptos teóricos asociados al pluralismo (82\%) y género (73\%). Se evidenció que en la comunidad existe discriminación por género para ejercer derechos por parte de las mujeres (82\%) y que hay desigualdad en el acceso a la educación (75\%) y oportunidades laborales (73\%). Se concluyó la vulneración de los derechos las mujeres indígenas Kichwa de la comunidad de Tocagón, ya que no pueden ejercerlos de acuerdo con el derecho convencional. Se recomienda capacitar en esta temática para lograr un ejercicio efectivo y positivo de estos derechos.

Palabras clave: Pluralismo jurídico, justicia indígena, derechos de las mujeres indígenas, derecho convencional.

\section{ABSTRACT}

The objective of this research work was to analyze from the Legal Pluralism, the rights of indigenous women of the community of Tocagón about Conventional Law. The research was based on a qualitative approach, of descriptive-documentary design. As an instrument for the collection of information, a survey-type questionnaire was designed, consisting of 11 questions with closed, dichotomous answers. It was applied to a sample of 75 indigenous Kichwa women from the Tocagón community, drawn from a population of 292. The analysis of the results showed that a high percentage of those surveyed are unaware of the existence of national and international norms related to the rights of women (65\%) and on theoretical concepts associated with pluralism (82\%) and gender (73\%). It was evidenced that in the community there is discrimination by gender to exercise rights by women (82\%) and that there is inequality in access to education (75\%) and job opportunities (73\%). It was concluded that there is a violation of the rights of indigenous Kichwa women in the community of Tocagón, which is why they cannot exercise them following conventional law. Training on this subject is recommended to achieve an effective and positive exercise of these rights.

Keywords: Legal Pluralism, Indigenous Justice, Indigenous Women's Rights, Conventional Law. 


\section{INTRODUCCIÓN}

En el mundo, distintas normas, resoluciones, disposiciones contienen los derechos de las mujeres y la protección de ellas. Derechos que son universalmente aplicados también a la mujer indígena y que según la Comisión Interamericana de Derechos Humanos - CIDH (2017) en el informe titulado: "Las Mujeres Indígenas y sus Derechos Humanos en las Américas", aun en la actualidad deben superar tropiezos para ejercerlos, tales como: dificultad para acceder al trabajo, a los servicios de salud, a la educación. Concluye el referido informe que "la exclusión política, social y económica de las mujeres indígenas contribuye a una situación permanente de discriminación estructural, que las vuelve particularmente susceptibles a diversos actos de violencia".

Los principios de paridad, igualdad, inclusión o participación se convierten en directrices que deben seguirse con el propósito de garantizar y respetar la actuación de la mujer indígena en su comunidad y en cualquier espacio o contexto social, económico, legal, laboral, etc. Sin embargo, la realidad es otra. En Ecuador, las comunidades mantienen y practican costumbres ancestrales, todo derivado de su propia naturaleza como pueblo indígena, elementos arraigados a su cotidianidad e interconectados con su idiosincrasia y cosmovisión.

De allí que, la finalidad de abordar esta temática socio jurídica que topa a las mujeres de la comunidad, surge desde el proyecto de investigación de la carrera de Derecho de la Universidad de Otavalo relacionado con el pluralismo jurídico a través de su línea investigativa vinculada con la Justicia Indígena y los derechos fundamentales y el trabajo de grado que se encuentra en el repositorio de la carrera de Derecho de la Universidad de Otavalo. La comunidad de Tocagón por ser una población indígena, su organización, funcionamiento y prácticas de vida se encuentran definidas por su realidad ancestral. En esta, así como en otras comunidades y pueblos de Ecuador su realidad se apega a sus creencias, su espiritualidad, su razón de vida a partir de sus costumbres. El comportamiento de hombres y mujeres deviene de su propia idiosincrasia cultural de sus antecesores, razón por la cual se hace necesario conocer, a partir del pluralismo jurídico, cómo las mujeres indígenas kichwa, de dicha comunidad ejercen sus derechos y si estos están en correspondencia con el derecho convencional.

En este sentido, como antecedentes académicos, se consideró la investigación titulada: Régimen jurídico de la participación de las mujeres indígenas dentro del Pluralismo Jurídico ecuatoriano, realizado por Cifuentes (2018) y cuyo objetivo fue realizar un diagnóstico del rol de la mujer en el ejercicio de la justicia indígena. A partir de un estudio teórico, se realizó un análisis del pluralismo jurídico y del género para concluir que las mujeres indígenas no entienden el concepto de igualdad como las mujeres mestizas o blancas, pues su interpretación es muy particular derivada de sus costumbres ancestrales, por lo que tampoco reconocen sus derechos.

En un contexto internacional, como primer antecedente se presenta el estudio realizado por Hayes (2016), titulado: "Pluralismo Jurídico en Bolivia". La coexistencia del derecho indígena y el derecho estatal en Bolivia. El estudio concluyó que la coexistencia de diversas culturas y etnias dentro de los Estados ha dado lugar a la consolidación y reconocimiento de la existencia del pluralismo jurídico. Los elementos conceptuales de este trabajo sirven para fundamentar teóricamente los elementos teóricos del pluralismo jurídico.

Por su parte, Serrano (2018), realizó una investigación titulada: "Agendas de las mujeres campesinas e indígenas en el proceso constituyente de Montecristi: Imposiciones, Concesiones y Olvidos, a partir de un análisis socio-jurídico de los factores de exclusión e inclusión y de la revisión de los discursos archivados en la Asamblea Constituyente"; se concluyó, que aún falta reconocer y reivindicar a estas mujeres en sus derechos, menoscabados a partir de relaciones de poder y del momento político vivido. Este trabajo aportó elementos para reconocer las actuaciones de las mujeres indígenas en la realidad política ecuatoriana. Los tres estudios antes mencionados, contribuyen a fortalecer los conceptos estructurales de la investigación.

Como antecedentes jurídicos, es necesario resaltar la Constitución de 2008 del Ecuador en su artículo 1 y 171 consagra la existencia de varios sistemas de Justicia dentro del territorio ecuatoriano. El fundamento jurídico de la investigación se encuentra establecido en los instrumentos jurídicos garantistas de derechos y de justicias aprobados por el gobierno de la República del Ecuador y cuya vigencia fortalecen la delimitación espacial del objeto de estudio, a saber: Convenio 169 de la Organización Internacional del Trabajo (1989), Declaración sobre los Derechos de los pueblos indígenas (2007), Convención sobre la eliminación de todas las formas de discriminación contra las mujeres (1979), Constitución de la República del Ecuador (2008), Ley Orgánica Integral para la prevención y erradicación de la violencia de genero contra las mujeres (2018).

El objetivo General de esta investigación recayó sobre el análisis de los derechos de las mujeres indígenas de la comunidad de Tocagón en relación con el Derecho Convencional desde el Pluralismo Jurídico. Para ello, se identificaron los elementos conceptuales del Pluralismo Jurídico con relación al derecho propio y las tradiciones ancestrales de las comunidades indígenas. Se determinaron los derechos de las mujeres indígenas Kichwa de la comunidad de Tocagón, desde una perspectiva de género y se estableció la correspondencia entre el ejercicio de sus derechos con el derecho convencional.

\section{MÉTODOS}

Esta investigación se orientó bajo un enfoque cualitativo, que según Ñaupas et al. (2014) "su propósito es reconstruir la realidad, descubrirlo, interpretarlo" pues permitió conocer el ejercicio de los derechos de las mujeres indígenas en su propia lengua Kichwa, y en el contexto de la vida cotidiana de la comunidad de Tocagón. Para esto, se empleó el método analítico que de acuerdo con Lopera et al. (2010) "es un camino para llegar a un resultado mediante la descomposición de un fenómeno en sus elementos constitutivos y así lograr analizar la realidad en estudio" (p.17) este análisis se sustentó en las encuestas aplicadas a las mujeres de la comunidad. En cuanto al diseño de la investigación fue no experimental y descriptivo que de acuerdo con Hurtado (2000) implica: "caracterizar globalmente el evento de estudio o enumerar sus características".

Asimismo, el tipo de investigación es el documental, en virtud que se realizó una revisión de documentos relacionados con el problema de investigación, como doctrina y normas contenidas en los cuerpos jurídicos internacionales y nacionales. Y dentro de las técnicas para la recolección de la información, siguiendo a Hurtado (2000), se usó "la observación para describir la situación en estudio"; la revisión documental para explicar los elementos teóricos apoyada en el análisis de contenido y como instrumento se elaboró un cuestionario tipo encuesta para conocer la realidad del ejercicio de los derechos de las mujeres indígenas de esta comunidad.

El cuestionario, como instrumento estuvo compuesto por once (11) preguntas cerradas, de respuestas dicotómicas (si/no) y se aplicó a una muestra de setenta y cinco (75) mujeres indígenas kichwa de la comunidad de Tocagón que fue extraída de una población de doscientas 
noventa y dos (292), cuyas edades estuvieron comprendidas entre los 21 y 50 años. Para verificar la validez de constructo del cuestionario este fue sometido a la validación de juicio de tres (3) expertos en pluralismo jurídico y género y, para determinar la confiabilidad de este, se aplicó una prueba piloto a una muestra parecida a la población bajo estudio, dando como resultado un alfa de Cronbach de 0,85 , lo que resultó altamente confiable.

Para la aplicación de este cuestionario se hizo necesario el traslado a dicha población y visitar a cada mujer en su vivienda, haciendo el uso de su propia lengua Kichwa a través de la investigadora Zoila Guamán Amaguaña quien es mujer kichwa Otavalo que habita en la Comunidad, todo ello para poder explicar el propósito del instrumento como parte de esta investigación, de acuerdo con una interpretación que respondiera a la interculturalidad y al enfoque de género que logró comprender el verdadero alcance de cada pregunta y de cada respuesta.

La comunidad de Tocagón, es una comunidad indígena localizada en la provincia de Imbabura, Cantón Otavalo, parroquia San Rafael que, según datos del Instituto Nacional de Estadística y Censos (2010), cuenta con una población de 400 familias kichwa Otavalo.

\section{RESULTADOS Y DISCUSIÓN}

\section{Los Derechos de las mujeres desde una perspectiva de Género}

La Declaración Universal de los Derechos Humanos de 1948 sobre la base de la libertad, la justicia y la paz reconoce los derechos en igualdad de condiciones e inalienables de todos los hombres. A partir de ella se aprueban el Pacto Internacional de Derechos Civiles y Políticos y el Pacto Internacional de Derechos Económicos, Sociales y Culturales que juntos conforman la Carta Internacional de Derechos Humanos. Todos estos documentos tienen carácter vinculante para aquellos Estados que los ratifican y permanentemente se elaboran recomendaciones para garantizar el cumplimiento de sus disposiciones.

De acuerdo con Carpizo (2011), "la dignidad humana es el fundamento del derecho internacional de los derechos humanos". Afirma el autor que dicho elemento es un:

Conjunto de atribuciones reconocidas en los instrumentos internacionales y en las Constituciones para hacer efectiva la idea de la dignidad de todas las personas y, en consecuencia, que puedan conducir una existencia realmente humana desde los ámbitos más diversos, los que se imbrican, como el individual, el social, el político, el económico y el cultural.

La Constitución de Montecristi, según Grijalva Jiménez (2011), "elimina las clasificaciones tradicionales de derechos. Lo hace con el propósito de enfatizar el carácter complementario y la igual jerarquía de todos los derechos constitucionales". "Incluso al referirse a los derechos colectivos los denomina "derechos de las comunidades, pueblos y nacionalidades", para destacar que también otros derechos pueden exigirse eventualmente de forma colectiva".

De igual forma, incorpora elementos innovadores como principios para la protección de los derechos humanos de las mujeres: el estado laico, la igualdad material y la paridad, según lo afirman Rosero y Goyes (2008).

Asimismo, regula la protección especial de niños, niñas, adolescentes, mujeres adultas, mujeres embarazadas y en período de lactancia, adul- tas mayores y desplazadas como posibles víctimas de maltrato, violencia doméstica o sexual y/o explotación sexual. Estas mismas garantías por supuesto son de aplicación igualitaria para las mujeres indígenas.

Cabe destacar el acceso a la educación como un derecho fundamental reconocido en el Pacto Internacional de Derechos Económicos, Sociales y Culturales (art. 13), la Convención sobre los Derechos del Niño (art. 28) y la Convención sobre la Eliminación de Todas las Formas de Discriminación contra la Mujer (art. 10.). Muchas han sido las situaciones que se han presentado en el menoscabo de este derecho, por lo que organismos internacionales han hecho hincapié en implementar estrategias que propendan a disfrutar de una educación universal y gratuita, donde se superen, principalmente, los obstáculos que afrontan niñas y mujeres "como el matrimonio precoz, los embarazos, el trabajo infantil y la violencia" (ONU, 2014).

De acuerdo con el Comité para la Eliminación de la Discriminación contra la Mujer, recomendación general N. ${ }^{\circ} 28$ (2010) relativa a las obligaciones básicas de los Estados parte, que de conformidad con el artículo 2 de la Convención sobre la Eliminación de Todas las Formas de Discriminación contra la Mujer, la equidad:

En algunas jurisdicciones... se utiliza para referirse al trato justo de la mujer y el hombre en función de sus necesidades respectivas. Esto puede incluir un trato igual, o un trato diferente pero considerado equivalente en cuanto a los derechos, los beneficios, las obligaciones y las oportunidades (párr. 22).

"El significado social y cultural que la sociedad atribuye a las diferencias biológicas da lugar a relaciones jerárquicas entre hombres y mujeres y a la distribución de facultades y derechos en favor del hombre y en detrimento de la mujer" (ONU, 2014). Asimismo, asevera la Organización de las Naciones Unidas (2014) en su informe que esta afirmación deriva en la marcada diferencia entre hombres y mujeres y, por supuesto, en el desigual valor que se le otorga a esta última en el ejercicio del derecho al trabajo, acceso al poder y a instancias de decisión, al manejo de la propiedad y los recursos, cuestiones que fundamentan la incorporación de la perspectiva de género como una estrategia y metodología que permita analizar desde esta visión como afecta esta discriminación a hombres y mujeres.

Es así como el, Comité para la Eliminación de la Discriminación Racial también abordó esta situación y en su recomendación general N. ${ }^{\circ} 25$ (2000), acerca de las dimensiones de la discriminación racial relacionadas con el género afirma que:

La discriminación racial no siempre afecta a las mujeres y a los hombres en igual medida ni de la misma manera. Existen circunstancias en que afecta únicamente 0 en primer lugar a las mujeres, 0 a las mujeres de distinta manera 0 en distinta medida que a los hombres.

De ello puede inferirse que el papel de la mujer sigue siendo afectado y vulnerados sus derechos, aun cuando en lo formal estos se encuentran enunciados, aprobados y en acciones, defendidos, en diferentes instrumentos internacionales y nacionales. Según la Comisión Interamericana de Mujeres (2015), la Convención Interamericana para Prevenir, Sancionar y Erradicar la Violencia contra la Mujer (Convención de Belém do Pará) "fue el primer tratado vinculante en el mundo en reconocer que la violencia contra las mujeres constituye una violación sancionable de derechos humanos". Entre los derechos de las mujeres destacan: 
Derecho a la vida pública y política, la salud, la educación, el trabajo, los derechos sexuales y reproductivos, el derecho a un nivel de vida digno, a la migración y el acceso a la justicia.

Desde esta perspectiva de género cabe mencionar que en Bolivia se encuentra vigente la Ley contra el racismo y toda forma de discriminación (2010) y en su artículo 5, literal e) define la equidad de género como:

Es el reconocimiento y valoración de las diferencias físicas y biológicas de mujeres y hombres, con el fin de alcanzar justicia social e igualdad de oportunidades que garantice el beneficio pleno de sus derechos sin perjuicio de su sexo en los ámbitos de la vida social, económica, política, cultural y familiar.

La Constitución Política del Estado Boliviano del 2009, también recoge en su artículo 15, el derecho de la mujer a no sufrir violencia física, sexual o psicológica, tanto en la familia como en la sociedad. Desde esta visión se establece que aun cuando existen diferencias entre sexos privilegia el ejercicio y goce de sus derechos en todos los contextos; situación que en la realidad pareciera verse afectada y que según Serrano (2019) muchos han sido los obstáculos que han encontrado las mujeres indígenas para superar la desigualdad y la opresión, elementos propios de sus realidades y sus propias culturas. En este orden de ideas, la Fundación Promoción y Desarrollo de la Mujer (PRODEMU) (2018) indica:

Importantes grupos de mujeres se han reunido en instancias nacionales demandando la necesidad de generar una agenda de largo plazo en materias tan relevantes que van desde derechos humanos, salud, educación, acceso a la justicia, medio ambiente y territorio, cultura, energía, desarrollo productivo; acceso a la política pública y representación política.

Estas situaciones de distinción clara, entre las formas de actuar de las mujeres y los derechos que ellas pueden ejercer en la realidad, ha conllevado a hacerse visible en su entorno más cercano, acciones de posible intimidación que las convierte en víctimas en sus propios espacios. Muchas de las mujeres en el mundo luchan para salir de estas situaciones y luchar por el pleno ejercicio de sus derechos. A lo que manifiesta Espinoza y Gallegos (2018) "la desigualdad entre los sexos es un fenómeno presente desde hace ya mucho tiempo y que se da en diversas culturas; incluso en algunos países la discriminación hacia la mujer empieza antes de su nacimiento".

En Ecuador existe un elevado número de mujeres indígenas que no se han incorporado a la educación básica, por ende, la mayoría de los pueblos indígenas mantienen su propio idioma que es el Kichwa; esta situación trae aparejada otra vulneración de derechos que se evidencia en algún tipo de violencia psicológica y patrimonial que según Berni (2018):

Sobrepasa el $50 \%$ en todas las provincias del país, en todos los niveles de instrucción y el más alto porcentaje se da en mujeres alfabetizadas $70 \%$, seguida por mujeres sin ninguna instrucción $66,9 \%$, en educación básica $64 \%$, educación media $8 \%$, educación superior 55,6\% y posgrado 52,8\%.

En las comunidades, ser mujer indígena conlleva un rol muy grande. En las responsabilidades de hombres y mujeres está primero la cultura materna, lo que significa que ambos cumplen sus funciones naturales y luego las funciones que les exige su género. Por ejemplo, es costumbre que al casarse el primer hijo tiene que ser un niño. Marrero y Lasso de la Vega (2016), al respecto afirma que en Ecuador:
La violencia de género hacia las mujeres se observaba tanto en el ámbito legislativo, en el ejecutivo y en el judicial, y en otros sectores de la sociedad. Ecuador no era la excepción, y la mujer que sufría violencia por parte de su pareja no podía denunciarla y menos aún exigir sanción para el agresor.

En este contexto, la Constitución ecuatoriana de 2008 consagra en su artículo 57, numeral 10:

Se reconoce y garantizará a las comunas, comunidades, pueblos y nacionalidades indígenas, de conformidad con la Constitución y con los pactos, convenios, declaraciones y demás instrumentos internacionales de derechos humanos, los siguientes derechos colectivos: 10. Crear, desarrollar, aplicar y practicar sus derechos propios o consuetudinarios, que no podrá vulnerar derechos constitucionales en particular de las mujeres, niñas, niños, y adolescentes.

De igual forma, la Constitución de la República del 2008 garantiza los derechos de las mujeres, en el Capítulo sexto, Sección tercera, título "Formas de trabajo y de retribución", artículo 331:

El Estado garantizará a las mujeres igualdad en el acceso al empleo, a la formación y promoción laboral y profesional, a la remuneración equitativa, y a la iniciativa de trabajo autónomo. Se adoptarán todas las medidas necesarias para eliminar las desigualdades. Se prohíbe toda forma de discriminación, acoso 0 acto de violencia de cualquier índole, sea directa o indirecta, que afecte a las mujeres en el trabajo (Constitución, 2008, p.155).

En la "Declaración Americana sobre los Derechos de los pueblos indígenas" (2016) se plantea: "promover los derechos de los pueblos indígenas en las Américas, así como brindar herramientas para una participación e incidencia eficaz y efectiva de líderes indígenas en espacios y procesos políticos a nivel interamericano, subregional, nacional y/o comunitario". En este instrumento internacional, se establece la igualdad entre hombre y mujer así: "Reconocer y proteger los derechos humanos de las mujeres indígenas, eliminando toda forma violencia y discriminación contra ellas, así como contra las niñas y niños indígenas".

Con relación al cuestionario, a la pregunta "¿Conoce usted qué es el pluralismo jurídico?" Se muestra que el $88 \%$ de las encuestadas respondieron en forma negativa, lo que significa que un alto porcentaje de las mujeres indígenas kichwa de la comunidad de Tocagón desconocen el concepto de pluralismo jurídico; mientras que el 12\% respondió positivamente. En cuanto a si ¿Sabe usted si la Constitución de 2008 reconoce la existencia de la justicia ordinaria y de la justicia indígena? Se evidencia que sólo el 15\% de las mujeres indígenas kichwa de la comunidad de Tocagón reconocen la existencia de la justica ordinaria y de la justicia indígena en la carta magna fundamental; en contraposición con el $82 \%$ restante que contestó no tener información sobre esto. A la interrogante ¿Usted tiene una idea clara sobre lo que es el género? El resultado obtenido señala que el $37 \%$ de las participantes contestaron que sí tienen una idea clara sobre lo que es género, mientras que el $63 \%$ afirmó no saberlo.

Ante la pregunta "¿Conoce usted normas internacionales que resguarden los derechos de las mujeres?" la respuesta arrojada por el 40\% de la población encuestada fue positiva; esto permite afirmar que 30 mujeres kichwa de la comunidad de Tocagón de una muestra de 75 dijeron que sí conocen normas internacionales que resguardan los derechos de las mujeres; mientras que el $60 \%$ contestó no conocerlas que corresponde a 45 sujetos participantes. "¿Conoce usted si a nivel internacional existen 
normas para proteger los derechos de las mujeres indígenas?" Los datos obtenidos indican que el 35\% de las mujeres kichwa de la comunidad de Tocagón, encuestadas en esta investigación respondieron que sí tienen conocimiento de que existen normas internacionales que protegen los derechos de las mujeres indígenas; en lugar del 65\% restante que contestó que no conoce de la existencia de esta normativa. En este aparte, se estima conveniente acotar que, durante la conversación, algunas mujeres indígenas kichwa de esta comunidad comentaron que aun cuando saben de la concurrencia de estas normas, las mismas no se aplican pues en este proceso chocan elementos culturales que lo impiden, lo que se conjuga con las prácticas ancestrales que se practican en la comunidad.

"A nivel de la comunidad de Tocagón, ¿cree usted que se respetan los derechos de las mujeres?" Se refleja que el $22 \%$ de las mujeres indígenas kichwa de la comunidad de Tocagón, participantes del estudio, indicaron que en esta población sí se respetan sus derechos, en lugar del $78 \%$ que contestó en forma negativa. "¿Considera usted que las oportunidades laborales entre hombres y mujeres son iguales en su comunidad?" Las respuestas indican que el $27 \%$ de las mujeres Kichwa de la comunidad de Tocagón, sí estima que hay igualdad de oportunidades de trabajo entre ambos sexos en esta población. A la pregunta "¿Considera usted que el derecho a la educación es igualitario para la mujer indígena en su comunidad?" se muestra que el 17\% de las mujeres indígenas kichwa de la comunidad de Tocagón, respondieron que sí considera que el ejercicio del derecho a la educación es igualitario; mientras que el 83\% contestó en forma negativa, lo que evidencia que un alto porcentaje de ellas considera un trato desigual que les impide acceder en igualdad de oportunidades a ejercer este derecho.

Con respecto a esta interrogante "¿Considera usted que la mujer indígena en su comunidad se siente discriminada al no lograr acceder a la educación?", la información indica que el 75\% de las mujeres kichwa encuestadas contestaron que sí se sienten discriminadas al no lograr acceder a la educación; en lugar de un 25\% de ellas que afirmaron no sentirse discriminadas. Finalmente, ante la pregunta “¿Considera Usted que los derechos reconocidos internacional y constitucionalmente en el Ecuador a las mujeres indígenas son aplicados en su familia y en la comunidad donde viven?" Se muestra que el $25 \%$ de las mujeres kichwa de la comunidad de Tocagón consideran que los derechos reconocidos internacional y constitucionalmente en el Ecuador sí son aplicados en su familia y en la comunidad; mientras que el $75 \%$ contestó lo contrario.

\section{El pluralismo jurídico}

La existencia en el mundo de diversas de pueblos y comunidades indígenas ha conllevado a modificar la visión monista del derecho a la concepción pluralista del mismo. Irigoyen (2004) al respecto afirma que: "En la última década del siglo XX emerge lo que podríamos llamar el horizonte pluralista", en este contexto el citado autor destaca: "el reconocimiento de los pueblos indígenas como sujetos políticos y no sólo como objetos de políticas que dictan otros"; y se "cuestionan dos herencias centrales de la juridicidad republicana: Ios binomios Estado-nación y Estado-derecho".

Estos elementos comienzan a definir una nueva posición más pluralista y diversa que se vislumbra en cambios constitucionales en varios países. Al respecto, refiere Sisa (2014) que el pluralismo jurídico en Ecuador:

Nace como resultado de la búsqueda de un Derecho que responda, de manera coherente, a la realidad social; misma que en nuestro país está compuesta por una gran diversidad de culturas y comunidades que la enriquecen permanentemente y, por consiguiente, deben ser consideradas y respetadas. La diversidad de pueblos y la coexistencia de los sistemas de estos con los sistemas jurídicos ordinarios conocidos a lo largo de la historia, conllevó a incorporar elementos para construir una concepción pluralista.

En este sentido, Santos (2018) afirma el nuevo pluralismo jurídico como:

La relación entre el sistema jurídico oficial y los otros órdenes que se articulan a él deja de ser vista como algo apartado o diferente y es abordada como una relación más compleja e interactiva, en la que se ve a la pluralidad jurídica como parte del campo social.

El componente social de esta afirmación deviene de la incorporación de las prácticas propias de las diferentes comunidades y pueblos indígenas, de la conformación y respeto de su territorio, del ejercicio de su justicia de manos del nombramiento y designación de sus propias autoridades. Estos constituyen, sin duda, la raíz fundamental de la aceptación de la diversidad de estas sociedades para lograr la convivencia en paz y armonía, lo que propende según Quiroz (2017) a reconocer específicamente "sus particulares procedimientos utilizados para resolver sus conflictos internos, conforme a sus costumbres; y, para entender de mejor manera su cosmovisión".

La visión de coexistencia de dos sistemas normativos bajo la cual se construye la definición de pluralismo jurídico no puede analizarse dejando de lado que el ejercicio de los derechos reconocidos implica también el reconocimiento de las prácticas ancestrales de los pueblos y nacionalidades indígenas, es decir, la aceptación del derecho propio de estos conglomerados sociales tratando de evitar la aplicación arbitraria de las normas y principios occidentales por sobre su cultura. Y este derecho propio no puede tampoco concebirse solo como una forma resolutoria de conflictos (elementos estrictos de la aplicación de la justicia indígena), sino como de aspectos en que se evidencia del ejercicio de sus derechos en la vida cotidiana de estos sujetos. Para el caso específico de los derechos de las mujeres de la Comunidad de Tocagón, aunque sus tradiciones ancestrales y su derecho consuetudinario es constitucionalmente reconocido, no es menos cierto que la convencionalidad de los derechos internacionales y constitucionales deben garantizarse en su materialidad para equilibrar asimetrías de derecho desde el enfoque de género. Al respecto, refiere Grijalva Jiménez (2011) que:

El Estado plurinacional significa, entre otras cosas, que en su territorio coexisten diversas culturas que el desarrollo no puede desconocer ni destruir, sino respetar y adoptar medidas que las impulsen y enriquezcan, de aquí que la Constitución coherentemente dispone que el desarrollo ha de "proteger y promover la diversidad cultural y respetar sus espacios de reproducción e intercambio; recuperar, preservar y acrecentar la memoria social y el patrimonio cultural" (arts. 276, numeral 7).

En este contexto, Acosta (2019) afirma que: "Tanto la interculturalidad como el pluralismo jurídico parten de una premisa fundamental: el respeto por la diferencia de manifestaciones y prácticas culturales, sociales y espirituales que difieren o no coindicen con aquellas reconocidas 0 validadas por la sociedad dominante" (p.42). En el caso en estudio, compartimos lo esgrimido bajo un enfoque de equilibrio y justicia entre el sistema ordinario y el indígena desde la unidad que brinda un Estado constitucional de derecho y de justicia. 


\section{CONCLUSIONES}

Desde el análisis teórico, se identificaron los elementos conceptuales del pluralismo jurídico y se asumen como la coexistencia de dos sistemas jurídicos, de acuerdo con lo planteado por de Santos (1987) también denominada la "Ecología de los saberes". Es así que a partir del análisis de los textos normativos vigentes que soportan la investigación se concluyó que la Constitución de Montecristi de 2008 reconoce el derecho de los pueblos y nacionalidades indígenas como miembros de una colectividad determinada con rasgos culturales, propio territorio social y coexistencia en un plano de la igualdad, respeto y unión sin menoscabar los derechos fundamentales y humanos de cada sujeto de derecho como la mujer kichwa Otavalo que hace vida en la comunidad de Tocagón.

Desde la perspectiva de género, los derechos de los hombres y mujeres deben ser reconocidos como intrínsecos de cada persona, sin distingo de sexo. Esto lleva a concluir que tanto las mujeres como los hombres indígenas deben ejercerlos sin ningún tipo de discriminación; específicamente los reconocidos como fundamentales, sin perder la esencia misma de la comunidad y su derecho ancestral; por el contrario, equilibrando ambas funciones en justicia, equilibrio y armonía social.

Se pudo evidenciar que, desde el derecho convencional, las mujeres gozan de un cúmulo de derechos reconocidos universalmente y que, en el caso de las mujeres indígenas de la comunidad de Tocagón, originariamente cuentan con derechos que se ejercen en sus propias comunidades y que, de alguna forma, deben ser respetados de acuerdo con su propia realidad cultural, tradiciones e idiosincrasia ante sus propias organizaciones indígenas. No obstante, se nota la ausencia de coordinación legislativa y acuerdos entre la coexistencia de ambas realidades jurídicas en la comunidad, para que se dimensionen realmente el ejercicio de estos derechos.

En los resultados de la investigación se denota que existen desigualdades por género hacia las mujeres kichwa de la comunidad de Tocagón; especialmente en el ejercicio de los derechos a la educación y al trabajo; ello permite contestar a la interrogante planteada en forma negativa en cuanto a la convencionalidad. En este particular, es necesario que el Estado desde los distintos enfoques instaure políticas públicas de coordinación entre lo convencional y lo ancestral.

En la comunidad de Tocagón, muchas de las mujeres indígenas kichwa, no tienen conocimiento de los derechos que poseen ni de las normas que las protegen en su ejercicio, derivado también del poco manejo teórico-conceptual de elementos clave que no son propios de su cultura. De allí que resulte necesario que la academia pueda socializar los derechos convencionales que desde la Juridicidad deben ser la base del ejercicio de derechos en equilibrio con sus tradiciones ancestrales.

\section{REFERENCIAS}

Acosta, J. (2019). Derechos humanos de los pueblos indígenas en clave de pluralismo jurídico e interculturalidad. ÁNFORA, 26(47), $37-$ 60. https://bit.ly/3ebP6ok

Asamblea Legislativa Plurinacional. (2009). Constitución Política del Estado. La Paz, Bolivia. https://bit.ly/3cxC3wq

Asamblea Constituyente (2008). Constitución de la República del Ecuador. Montecristi, Quito: Asamblea Constituyente. https://bit. ly/3frPos0
Asamblea General de las Naciones Unidas (1979). Convención sobre la Eliminación de todas Formas de Discriminación contra la mujer. https://bit.ly/3cBivHD

Asamblea General de Naciones Unidas. (2005). Convención sobre la Eliminación de Todas las Formas de Discriminación contra la Mujer. https://bit.ly/31ulmvt

Asamblea Legislativa Plurinacional. (2010). Ley No. 045. Ley contra el racismo y toda forma de discriminación. Gaceta Oficial del Estado Plurinacional de Bolivia. La Paz, Bolivia. https://bit.ly/3sDc9wS

Asamblea Nacional del Ecuador. (2018). Ley Orgánica Integral para la Prevención y Erradicación de la violencia de género contra las mujeres. Quito: Ecuador. https://bit.ly/3cxNkwW

Berni, M. (2018). Estrativismo social machista en Ecuador. Violencia de género, femicidio. Revista Conrado 14, (61), 111-115. https:// bit.ly/3dfXoKc

Carpizo, J. (2011). Los Derechos Humanos: Naturaleza, denominación, características. Revista Mexicana de Derecho Constitucional. 25, julio-diciembre 2011. https://bit.ly/3sNPTAo

Cifuentes, A. (2018). Régimen Jurídico de la participación Mujeres Indígenas dentro del Pluralismo Jurídico Ecuatoriano. (Tesis de Grado). Pontificia Universidad Católica del Ecuador. Quito. Ecuador. https://bit.ly/2QHReuu

Comisión Interamericana de Derechos Humanos. (2017). Las mujeres indigenas y sus derechos humanos en las Américas. Dinamarca. Publicaciones IWGIA. https://bit.ly/3sEQHri

Comité para la Eliminación de la Discriminación Racial. (2000). Recomendación General No. 25. Ginebra, Suiza. https://bit. Iy/3u7EIZ6

Organización de Los Estados Americanos (OEA). (1994). Convención Interamericana para Prevenir, Sancionar y Erradicar la Violencia contra la Mujer. Belén Do Para. Brasil. https://bit.ly/3frOouM

Organización de las Naciones Unidas (ONU). (2007). Declaración de las Naciones Unidas sobre los Derechos de los Pueblos indigenas. Nueva York, Estados Unidos de Norteamérica. https:// bit.ly/31wNyhr

Espinoza, M; Gallegos, D. (2018). Discriminación Laboral en Ecuador. Revista Espacio. Caracas, Venezuela. https://bit.ly/3sBVdad

Guamán, Z. (2020). Los derechos de las mujeres indígenas de la comunidad de Tocagón desde el pluralismo jurídico. (Tesis de Grado). Repositorio de la Universidad de Otavalo, Ecuador. https://bit. Iy/3m2Cbr4

Grijalva, A. (2011). Constitucionalismo en Ecuador. Pensamiento Jurídico contemporáneo. Corte Constitucional para el período transición. Quito. Ecuador. https://bit.ly/2QHUKFc

Hayes, M. (2016). Pluralismo jurídico en Bolivia. La coexistencia del Derecho indígena y el Derecho estatal. (Tesis Doctoral). Universitat de Valencia, España. https://bit.ly/39rqQeR

Hurtado, J. (2000). Metodología de la investigación holística. Caracas, Venezuela: Fundación SYPAL. https://bit.ly/3fuPnnc

Instituto Nacional de Estadística y Censos (2010). Censo de población. Quito, Ecuador: Instituto Nacional de Estadística y Censos.

Irigoyen, R. (2004). Pluralismo Jurídico. Derecho Indígena y Jurisdicción especial en los países andinos. El otro Derecho, número 30, junio. ILSA, Bogotá D.C., Colombia. https://bit.ly/39qwy0n

Lopera, J., Ramírez, C., Zuluaga, C., Aristazábal, M., Ortiz, J. (2010). El método analítico como método natural. Nómadas. Critical Journal of Social and Juridical Sciences. 25, núm. 1. Euro-Mediterranean University Institute. Roma, Italia. https://bit.ly/3cBLx9S 
Marrero, 0., Lasso de la Vega, M. (2016). El tratamiento a la noticia sobre violencia de género a la mujer en los medios de comunicación en Ecuador. Su responsabilidad social. Revista Científica ECOCIENCIA. 3, (5). https://bit.ly/3w9PhqZ

Ñaupas, H., Mejía, E., Novoa, E., Villagomez, A. (2014). Metodología de la investigación cualitativa - cuantitativa y Redacción de la tesis. Bogotá, Colombia: Ediciones de la U. https://bit.ly/3aW2JWm

Organización de las Naciones Unidas (1948). Declaración Universal de los Derechos Humanos. Ginebra, Suiza. https://bit.ly/3sAUiXn

Organización Internacional de Trabajo (1989). Convenio 169. https://bit. ly/3sD5KC3

Organización de las Naciones Unidas (2014). Los derechos de las mujeres son derechos humanos. https://bit.ly/3cA1TQH

Organización de Estados Americanos, OEA. (2016). Declaración Americana sobre los Derechos de los pueblos indígenas. https://bit. ly/3uboBoo

Organización de las Naciones Unidas (ONU). (2010). Pacto Internacional de Derechos Económicos, Sociales y Culturales. Nueva York, Estados Unidos de América. https://bit.ly/31vEh9c

Promoción para el desarrollo de las mujeres. (PRODEMU). (2018). El derecho de las mujeres indigenas. Chile. https://bit.ly/3diulpg

Quiroz, E. (2017). Pluralismo Jurídico y Justicia indígena en Ecuador. Universidad Internacional del Ecuador. INNOVA Research Journal. 2, (12) 49-58. D0l: https://bit.ly/2Pc3gMA

Rosero Garcés, Rocío; Goyes Quelal, Solanda. Los derechos de las mujeres en la constitución del 2008 (Políticas Públicas). La Tendencia. Revista de Análisis Político. Referéndum: convergencia progresista. Quito: FES-ILDIS, (no. 8, octubre-noviembre 2008): pp. 77-82. ISSN: 13902571. https://bit.ly/2PaqBOF

Serrano, P. (2018). Agenda de las mujeres campesinas e indígenas en el proceso constituyente de Montecristi imposiciones concesiones y olvido. (Tesis de Grado). Universidad de Azuay, Ecuador. https://bit.ly/31y3tMA

Santos, B. (2018). Construyendo las Epistemologías del Sur. Buenos Aires. Argentina: CLACSO. https://bit.ly/3wbIDSa

Sisa, W. (2014). La Vulnerabilidad de los Derechos Humanos de la Justicia Indígena Ecuatoriana, con referencia al pueblo de pilahuin de la Provincia de Tungurahua en los dos últimos años. (Tesis de Grado). Universidad Central del Ecuador. https://bit.ly/3w5fiHY 